\title{
In vivo dosimetry in brachytherapy
}

Tanderup, Kari; Beddar, Sam; Andersen, Claus Erik; Kertzscher Schwencke, Gustavo Adolfo Vladimir; Cygler, Joanna E.

Published in:

Medical Physics

Link to article, DOI:

10.1118/1.4810943

Publication date:

2013

Document Version

Publisher's PDF, also known as Version of record

Link back to DTU Orbit

Citation (APA):

Tanderup, K., Beddar, S., Andersen, C. E., Kertzscher Schwencke, G. A. V., \& Cygler, J. E. (2013). In vivo dosimetry in brachytherapy. Medical Physics, 40(7), [070902]. https://doi.org/10.1118/1.4810943

\section{General rights}

Copyright and moral rights for the publications made accessible in the public portal are retained by the authors and/or other copyright owners and it is a condition of accessing publications that users recognise and abide by the legal requirements associated with these rights.

- Users may download and print one copy of any publication from the public portal for the purpose of private study or research.

- You may not further distribute the material or use it for any profit-making activity or commercial gain

- You may freely distribute the URL identifying the publication in the public portal

If you believe that this document breaches copyright please contact us providing details, and we will remove access to the work immediately and investigate your claim 


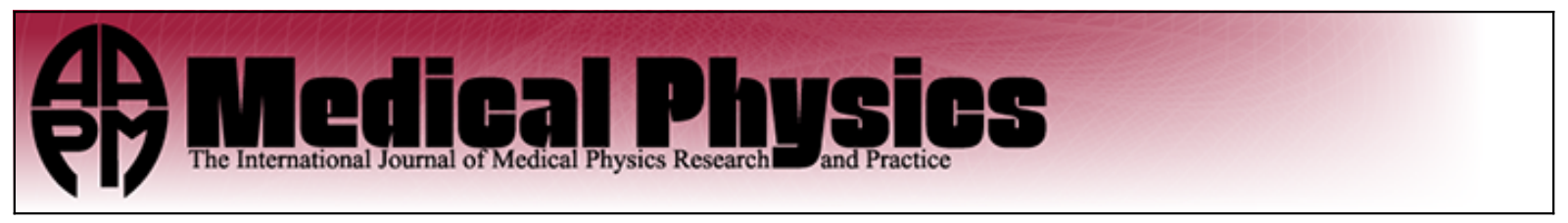

\section{In vivo dosimetry in brachytherapy}

Kari Tanderup, Sam Beddar, Claus E. Andersen, Gustavo Kertzscher, and Joanna E. Cygler

Citation: Medical Physics 40, 070902 (2013); doi: 10.1118/1.4810943

View online: http://dx.doi.org/10.1118/1.4810943

View Table of Contents: http://scitation.aip.org/content/aapm/journal/medphys/40/7?ver=pdfcov

Published by the American Association of Physicists in Medicine

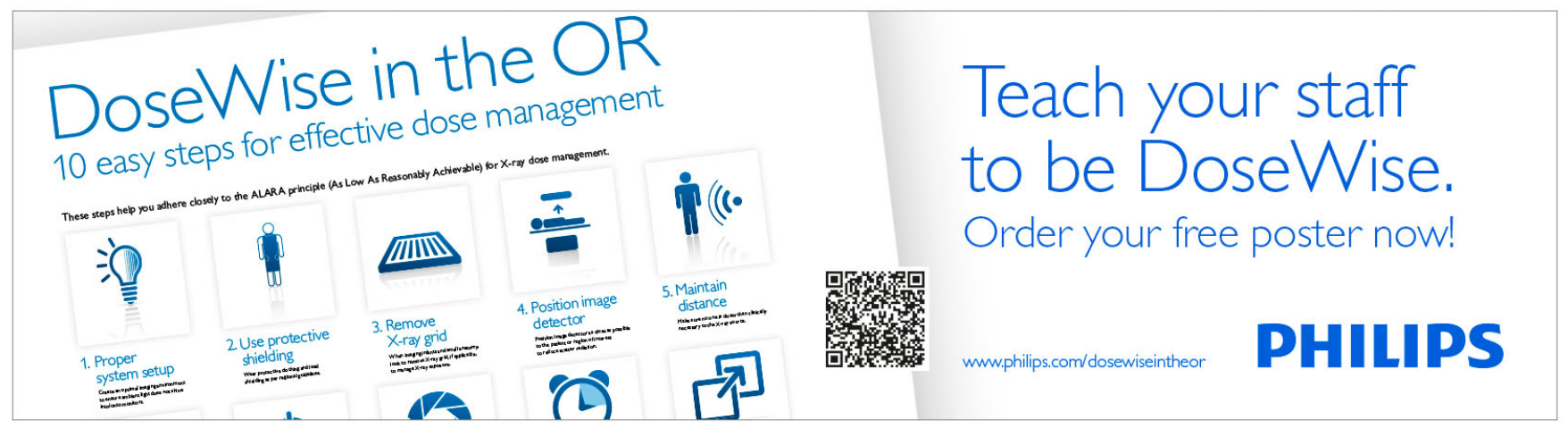




\title{
In vivo dosimetry in brachytherapy
}

\author{
Kari Tanderup ${ }^{a)}$ \\ Department of Oncology, Aarhus University Hospital, Aarhus 8000, Denmark and Department of Clinical \\ Medicine, Aarhus University, Aarhus 8000, Denmark
}

\author{
Sam Beddar \\ Department of Radiation Oncology, The University of Texas MD Anderson Cancer Center, Houston, \\ Texas 77030 \\ Claus E. Andersen and Gustavo Kertzscher \\ Center of Nuclear Technologies, Technical University of Denmark, Roskilde 4000, Denmark \\ Joanna E. Cygler \\ Department of Physics, The Ottawa Hospital Cancer Centre, Ottawa, Ontario K1H 8L6, Canada
}

(Received 15 January 2013; revised 12 April 2013; accepted for publication 16 April 2013; published 25 June 2013)

In vivo dosimetry (IVD) has been used in brachytherapy (BT) for decades with a number of different detectors and measurement technologies. However, IVD in BT has been subject to certain difficulties and complexities, in particular due to challenges of the high-gradient BT dose distribution and the large range of dose and dose rate. Due to these challenges, the sensitivity and specificity toward error detection has been limited, and IVD has mainly been restricted to detection of gross errors. Given these factors, routine use of IVD is currently limited in many departments. Although the impact of potential errors may be detrimental since treatments are typically administered in large fractions and with high-gradient-dose-distributions, BT is usually delivered without independent verification of the treatment delivery. This Vision 20/20 paper encourages improvements within BT safety by developments of IVD into an effective method of independent treatment verification. (C) 2013 American Association of Physicists in Medicine. [http://dx.doi.org/10.1118/1.4810943]

Key words: in vivo dosimetry, brachytherapy, treatment errors, quality assurance

\section{INTRODUCTION}

In vivo dosimetry (IVD) has been used in brachytherapy (BT) for decades and has been referred to in International Commission on Radiation Units and Measurements (ICRU) recommendations. ${ }^{1}$ The initial motivation for performing IVD in BT was mainly to assess doses to organs at risk (OAR) by direct measurements, because precise evaluation of OAR doses was difficult without 3D dose treatment planning. With the introduction of $3 \mathrm{D}$ image-guided $\mathrm{BT}$, it is now possible to calculate 3D tumor and OAR doses as well as dose volume histogram (DVH) parameters by the treatment planning system (TPS). ${ }^{2-4}$ Recording and reporting of dose based on 3D images is an important step forward. Current 3D image-based dose calculations are often more accurate for assessments of OAR doses than IVD measurements. However, there are situations with significant dose calculation uncertainties, in particular for low-energy photon-emitting sources and in heterogeneous media, where IVD may contribute to more precise dose reporting. ${ }^{5}$ Furthermore, potential organ movement in between imaging and treatment contributes to uncertainties in delivered dose, and IVD may have a potential role for identification of organ or applicator movements.

The complexity of BT has increased with the introduction of remote afterloading and 3D image-based dose planning. However, independent and patient specific quality assurance (QA) of dose delivery is not performed systematically, despite the risk of errors during the many manual steps involved in treatment planning and delivery. Furthermore, BT is typically administered with large doses per fraction ( $>5 \mathrm{~Gy}$ ), which means that the consequence of a fractional error may be substantial when compared to typical external-beam radiotherapy (EBRT) with delivery of smaller doses per fraction (e.g., 2 Gy). From this perspective, IVD is very relevant in BT as an independent method for detection of deviations or errors. ${ }^{6}$ Unfortunately, the interest has suffered much from difficulties and uncertainties of dose measurement, in particular the challenges of precise detector positioning in the high dose gradient fields. Error detection sensitivity and specificity of current systems has been compromised by such uncertainties, and therefore it seems that current IVD is only able to detect gross errors (Sec. IV.C), which happen less frequently. Furthermore, there has been a lack of good infrastructure in terms of commercially available dosimetry systems with straightforward procedures, which do not require extensive manpower and expertise. Substantial progress is needed in dose measurement methodology and infrastructure in order to bring IVD to its full potential.

Whereas IVD in BT needs further development to become well integrated into routine clinical practice, the situation is different in EBRT where IVD is more widely used and accepted as an independent check of dose delivery. ${ }^{7,8}$ Conformal EBRT is characterized by flat dose profiles and homogeneous dose in the target region. Therefore, conformal EBRT IVD for target dose verification has not suffered from the particular challenge typical for BT, which is detector positioning 
in high gradient fields. However, due to the heterogeneous fluence profiles and dose gradients, ${ }^{9}$ IVD in IMRT and VMAT for dose assessments in OARs, for instance, would involve some of the same challenges as are seen with BT. The energy dependence of detectors is often more crucial in BT as compared to EBRT because of the lower photon energy applied in BT. However, most small detectors that are used for IVD in EBRT, ${ }^{7}$ are in principle also suitable in BT. Transmission IVD based on, e.g., EPID has much potential for EBRT, ${ }^{10}$ but has not yet been much exploited for BT. In the accompanying Vision 20/20 paper, ${ }^{11}$ the current and future justifications of performing external beam IVD are discussed, and a comprehensive review of detectors used for IVD is provided.

The purpose of the current Vision 20/20 paper is to discuss current and future justifications of IVD for BT, and to identify current challenges that need to be addressed in order to advance the field over the next decade.

\section{JUSTIFICATION FOR IN VIVO DOSIMETRY}

\section{II.A. Errors and treatment variations in brachytherapy}

There is currently no systematic overview of the type and frequency of errors which occur in BT. Existing reports of BT accidents and errors are mainly based on retrospective event collection since prospectively structured investigations have not yet been performed on a multicenter scale. Furthermore, since current IVD systems suffer from unknown or poor sensitivity and/or specificity (see below), there are an unknown number of BT errors that remain undetected.

The International Commission on Radiological Protection (ICRP) Report Nos. 86 (Ref. 12) and 97 (Ref. 13) as well as IAEA safety report series 17 (Ref. 14) describe errors that have occurred in BT. BT errors and accidents are mainly related to human errors, although some errors are caused by mechanical events related to malfunction of the equipment. Examples of human errors are incorrect medical indication, source strength, patient identification, diagnosis or site of treatment, prescription, data entry, catheter, or applicator. High dose-rate (HDR) and pulsed dose-rate (PDR) afterloading techniques may introduce source positioning errors which are related to procedures specific for afterloading dose planning and treatment delivery. Source positioning errors may also occur in low dose-rate (LDR) manual loading procedures due to manual misplacement or source migration after the placement.

Afterloading BT embraces a variety of approaches to treatment planning, each involving specific error scenarios. Treatment planning can be performed without the use of a TPS by directly defining the target from x-ray image or clinical findings and applying a standardized or manually optimized treatment plan. An example would be an esophagus treatment where the treatment length, source offset, step size, and dwell times may be directly defined on site in some clinics and followed by a more or less manual entry of treatment parameters into the afterloader control software. For this scenario, the risk of errors is mainly related to the definition and manual entry of parameters. A number of manual steps are avoided with 3D image-based treatment planning. However, other sources of potential errors are introduced in this procedure. With 3D image-based dose planning, both target and source catheters are defined with imaging modalities such as ultrasound (US), magnetic resonance imaging (MRI), or computed tomography (CT). Mistakes or uncertainties in identification of source catheters or definition of applicator length can lead to source positioning errors. Dose optimization may involve a high degree of dwell time modulation, which means that the consequence of source positioning errors can lead to significant errors throughout the volumetric dose distribution. The treatment plan is usually directly exported from the treatment planning system to the treatment control station (TCS) and the afterloader. The automatic transfer reduces the risk of incorrectly entered parameters, although there is still a risk that a wrong plan is exported or imported. After treatment plan transfer, the afterloader is connected to the applicators; this process involves a risk of misconnection of the source transfer guide tubes.

Organ or applicator movement in between imaging and treatment represents a limitation on the accuracy of dose delivery unless imaging is performed immediately before delivery in a BT suite. For prostate BT, there have been reports of needle movements, ${ }^{15,16}$ and for gynecological BT, organ movement has been seen as well as occasional relocation of the applicator. ${ }^{17}$ IVD may be a tool for detection of such displacements and movements. However, direct inspection of the applicator (e.g., measurement of free needle length in prostate BT) or reimaging directly before treatment delivery may be an alternative way to validate that the dose delivery and dose recording are appropriate. ${ }^{18}$ Neither the use of repeated imaging nor IVD has demonstrated wide practical application for detection of organ and applicator movement. The conditions under which each of these may be practical and/or precise enough for use in clinical practice still remain to be clarified.

Certain errors can be identified by the afterloader safety systems which include builtin dummy check sources, a radiation detector, and source cable length control. ${ }^{19}$ However, afterloader safety systems are only able to detect a subset of all possible errors that may occur. Additional manual safety checks of the treatment planning and delivery procedure may be introduced such as verification of applicator reconstruction or source guide tube connection by a second observer. In addition, as documented in ICRP and IAEA reports, ${ }^{12-14}$ there are a number of errors that are not detected by safety systems or manual checks. IVD has the potential to complement other QA and quality control (QC) procedures, as part of the greater process of quality management, by providing an independent overall verification of the treatment delivery (Table I).

\section{II.B. Recording of dose in individual patients}

The increasing use of image-guided BT has been a huge step forward for the calculation and recording of tumor and OAR doses. In most cases, dose calculation is more accurate than IVD, mainly due to the challenge of precise detector positioning to measure the specific OAR or tumor doses (see, 
TABLE I. QA and detection of errors and variations occurring with afterloading BT.

\begin{tabular}{|c|c|c|}
\hline Quality item & Typical quality test & Role of in vivo dosimetry \\
\hline Source calibration & Independent source calibration in the department & $\begin{array}{l}\text { Minor importance, but may in certain situations } \\
\text { reveal calibration errors (if the in vivo dosimeter } \\
\text { calibration is independent from the actual } \\
\text { source). }\end{array}$ \\
\hline $\begin{array}{l}\text { Afterloader source positioning and dwell time } \\
\text { (nonpatient specific) }\end{array}$ & $\begin{array}{l}\text { Autoradiography, commissioning of applicators, } \\
\text { other source stepping and dwell time QA }\end{array}$ & $\begin{array}{l}\text { Minor importance, but may in certain situations } \\
\text { reveal errors which have not been identified with } \\
\text { standard nonpatient specific QA. }\end{array}$ \\
\hline Afterloader malfunction & $\begin{array}{l}\text { Unpredictable afterloader malfunction is } \\
\text { difficult to target with general QA. }\end{array}$ & $\begin{array}{l}\text { Major relevance, since afterloader malfunction } \\
\text { may have significant impact on dose, and IVD } \\
\text { has good potential to reveal such errors. }\end{array}$ \\
\hline Patient identification & Manual check & Not relevant \\
\hline Correct treatment plan & Manual check & $\begin{array}{l}\text { Relevant since it may detect if a wrong plan is } \\
\text { used for treatment, if DICOM transports to the } \\
\text { afterloader and dosimeter software are } \\
\text { independent. }\end{array}$ \\
\hline $\begin{array}{l}\text { Intra- and interfraction organ/applicator } \\
\text { movement }\end{array}$ & $\begin{array}{l}\text { Reimaging performed just before treatment } \\
\text { delivery can in some cases be used to assess } \\
\text { organ or tumor dose in image-guided BT. }\end{array}$ & $\begin{array}{l}\text { The relevance depends on detector positioning. } \\
\text { If the detector is fixed to the applicator, it will } \\
\text { not reveal applicator movement, contrary to } \\
\text { measurements in, e.g., urethra. For the organ } \\
\text { dose change detection, there are significant } \\
\text { challenges to position the detector precisely } \\
\text { (reimaging may be preferred). }\end{array}$ \\
\hline Applicator reconstruction and fusion errors & Manual check & $\begin{array}{l}\text { Relevant. Can detect applicator reconstruction } \\
\text { errors when the dosimeter is placed in an } \\
\text { optimal geometry. }\end{array}$ \\
\hline Applicator length/source-indexer length & Manual check & $\begin{array}{l}\text { Major relevance, since "source-indexer length" } \\
\text { errors may have significant impact on dose, and } \\
\text { real time IVD has good potential to reveal such } \\
\text { errors. }\end{array}$ \\
\hline Source step size (patient specific) & Manual check & $\begin{array}{l}\text { Major relevance if source step size is entered } \\
\text { manually. Otherwise the appropriate method to } \\
\text { check source stepping is part of nonpatient } \\
\text { specific QA. }\end{array}$ \\
\hline Interchanged guide tubes & Manual check & $\begin{array}{l}\text { Major relevance, since interchanged guide tubes } \\
\text { may have significant impact on dose, and IVD } \\
\text { has good potential to reveal such errors. }\end{array}$ \\
\hline Recording of dose & $\begin{array}{l}\text { General QA related to dose calculation in the } \\
\text { TPS }\end{array}$ & $\begin{array}{l}\text { Relevant when dose recording is not calculated } \\
\text { with sufficient accuracy by 3D image based TPS } \\
\text { calculation (e.g., lack of inhomogeneity or } \\
\text { radiation scatter corrections). }\end{array}$ \\
\hline
\end{tabular}

e.g., Sec. IV.C). However, for certain clinical situations TPS based dose calculations are currently associated with systematic deviations due to lack of corrections for heterogeneities and scatter conditions. ${ }^{20}$ This is particularly true for low energy sources such as ${ }^{125} \mathrm{I}$ or ${ }^{103} \mathrm{Pd}$ used in permanent implants of prostate, lung, and recently breast. ${ }^{21-23}$

Some dosimetry systems define specific points or volumes for dose reporting for individual patients such as the Paris System for interstitial implants; ${ }^{24}$ ICRU $38,{ }^{1}$ GEC European Society for Radiotherapy and Oncology (ESTRO), ${ }^{2}$ and ABS for cervix BT; ${ }^{.25}, 26$ as well as ABS (Ref. 27) and GEC ESTRO (Refs. 28 and 29) for prostate implants. This kind of reporting is important in treatment outcome assessments. However, the reporting is mostly based on treatment planning identification of such points/volumes. In the situation where the tumor and OARs move and/or get deformed, as is the case during permanent prostate implants, IVD can provide additional information about the dose delivered to the tumor and/or OAR for the given patient. In particular, for LDR and PDR treatments that involve long irradiation times, there may be anatomical changes such as prostate swelling, applicator movement, and organ movement, and IVD may be used to monitor the treatment progression. There have been studies of the point or segment dose measurements in rectum or urethra for gynecological ${ }^{30,31}$ or prostate implants ${ }^{32-34}$ or in the tumor. ${ }^{35,36}$ Some results confirm the agreement between the calculated and delivered doses, while others point to discrepancies between them. The reasons for these disagreements are not always clear and underscore the technical difficulties faced by IVD in BT. 


\section{CURRENT CLINICAL PRACTICE}

Typical sites for IVD are rectum, ${ }^{37-40}$ and bladder or urethra. ${ }^{32,33,41}$ Noninvasive detector positioning far from the source, e.g., on the skin, make the measurements insensitive to error detection because of low signal and difficulties to know precisely the relation between the source(s) and detector(s). IVD on skin is of relevance during the treatment of breast cancer ${ }^{42-44}$ since the skin is an OAR close to the treated volume and has significant dose calculation uncertainties. ${ }^{45}$

The practice of using IVD varies considerably among countries. In most countries, systematic use of IVD is not often incorporated as a standard QA or QC procedure. However, there is specific legislation in a number of countries making IVD obligatory. For example, France recently introduced a law that requires some form of IVD be performed on all EBRT patients. ${ }^{46}$ Unfortunately, there are no reports available about the value and benefit of routine BT IVD in these countries. In patterns-of-care studies from Europe and Latin America, it has been difficult to collect responses that homogeneously represent all countries. ${ }^{47,48}$ However, it seems that IVD is available in many institutions because it is reported to be used by $20 \%-33 \%$ of the centers. ${ }^{47-49}$ However, it is not further described how often, in which disease sites, or in how many patients. A recent survey by Sawakuchi et al. ${ }^{103}$ on Canadian cancer clinics showed that most of the clinics perform some form of IVD for special EBRT techniques. However, no IVD is performed routinely for BT treatments in Canada. A Japanese patterns-of-care study of cervix cancer reports that rectal and bladder IVD was used in $27 \%$ and $3 \%$ of the BT patients, respectively. ${ }^{50}$ IVD was carried out in $1.2 \%$ U.S. centers that performed vaginal BT in endometrial cancer patients. ${ }^{51}$ From available literature and institutional reports, it seems that IVD most often appears as diode rectal dosimetry performed during intracavitary BT in gynecological cancer. There are a number of groups reporting institutional use of IVD in prostate cancer, but there are no general reviews or reports that indicate this technique is commonly used, or what was the observed error rate.

\section{CHALLENGES SPECIFIC TO IN VIVO DOSIMETRY IN BRACHYTHERAPY}

\section{IV.A. High dose gradients and detector positioning}

The BT dose distributions are characterized by steep dose gradients that depend strongly on the distance from the detector to the source. At a distance of $4 \mathrm{~mm}$ from a linear source, the dose gradient is approximately $50 \% / \mathrm{mm}$. The gradient decreases to around $6 \% / \mathrm{mm}$ and $5 \% / \mathrm{mm}$ at distances of 20 and $35 \mathrm{~mm}$, respectively. Therefore, even small uncertainties in detector positioning may lead to significant uncertainties in dose measurements. The ability of IVD to detect errors depends critically on where the detectors can be placed and how well these detector positions are known. The optimal region for detector placement balances the favorable signal-to-noise ratio (SNR) with the smallest possible impact of positional uncertainties. ${ }^{35,36}$ This optimal region is different and specific for each detector and patient category due to differences in positional uncertainties of the detector as well as sensitivity, energy dependence, and angular dependence of the detector response.

The steep dose gradient induces additional challenges when IVD is used for individual measurement of organ dose. The dosimeter needs to be positioned in a clinically relevant region, and for the bladder and rectum, it is a major difficulty to establish a stable dosimeter position directly adjacent to the most exposed part of the organ wall. ${ }^{52}$ The anatomy of the urethra is more favorable for performing accurate IVD in prostate BT since this organ is smaller and less deformable. Several challenges regarding optimal dosimeter positioning could be solved with dedicated clinical components, e.g., urethral catheters and rectal or bladder balloons, that would both stabilize and offer accessibility at desired dosimetry sites (see Sec. VI.A).

Ease of positioning as well as good detector stability can be obtained by insertion of extra invasive catheters or needles. However, the potential use of such invasive procedures raises ethical issues because of accompanying risks for the patient such as infection. The extra procedure must be of clear benefit to the patient and since IVD still has to demonstrate that it adds significant improvement in terms of safety and quality there is currently reluctance among clinicians to introduce extra invasive procedures.

\section{IV.B. Energy dependence of detectors}

Another major challenge for IVD in BT is the energy dependence (relative absorber dose sensitivity) (Ref. 53) of existing detectors in the intermediate-to-the-low energy range of the photon or gamma energy spectra that are commonly encountered $\left({ }^{60} \mathrm{Co},{ }^{137} \mathrm{Cs}\right.$ down to $\left.{ }^{103} \mathrm{Pd}\right)$ covering $>1$ to $<0.03$ $\mathrm{MeV}$ photons. ${ }^{54}$ The energy dependence of a detector is defined as the dependence of the detector reading as a function of the absorbed dose in water on the photon or electron energy spectrum. Energy dependence has implications both for calibration of the dosimeter and for conversion of detector reading into measured dose during patient measurements. Most commonly used detectors exhibit a higher energy-dependence in BT than in megavoltage beams, because the photoelectric effect causes an over-response in the BT energy range. This is due to the fact that detector response depends more on the mass-attenuation absorption coefficients (large cavities) than on the mass-collisional stopping powers (small cavities: satisfying the Bragg-Gray conditions). Therefore, the energy dependence (absorbed dose sensitivity) needs to be determined for the type of detector used for the specific radioactive source since the energy spectrum depends also on source model (especially for low-energy sources). The absorbed dose sensitivity must be corrected for if the source is calibrated with a radiation source different from the BT source. Even more challenging is that the detector response may change with depth in tissue from the source because the BT photon energy spectrum changes as function of source distance. 
TABLE II. Treatment error detection and definition of specificity and sensitivity.

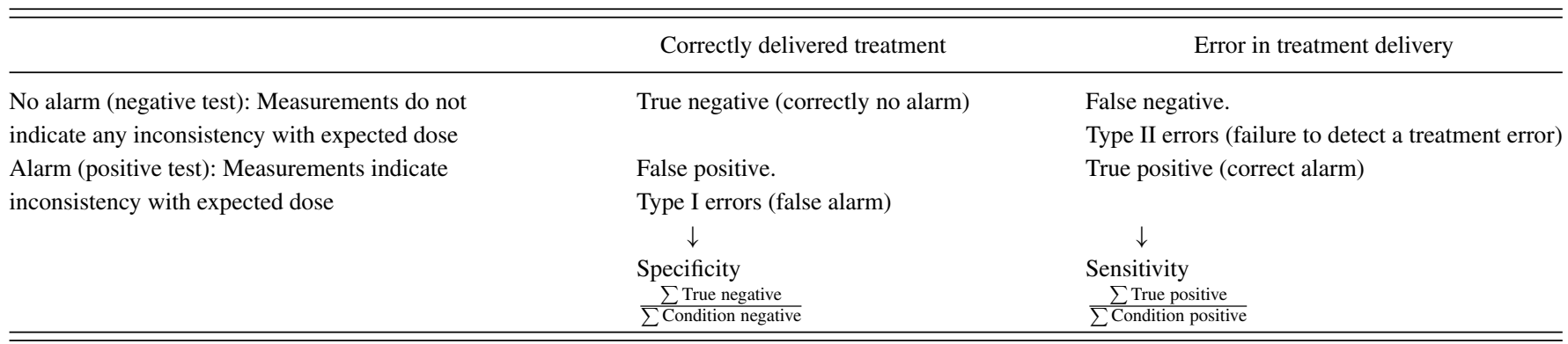

\section{IV.C. Sensitivity and specificity}

Sensitivity and specificity are statistical measures of the performance of a binary test such as the classification of an in vivo measurement into "No alarm" or "Alarm" (Table II). The sensitivity measures the proportion of actual positives which are correctly identified as such (e.g., the percent of treatment errors which are correctly identified as errors). Specificity measures the proportion of negatives which are correctly identified (e.g., the percentage of correct treatments which are correctly identified as not being affected by errors). These two measures are closely related to the concepts of type I (false alarm) and type II (lack of error detection) errors. Type I and II errors are statistical concepts and should not be mixed up with "treatment error." For instance, a type I error would indicate an alarm during a correctly administered treatment, hence indicating "false alarm." A theoretical, optimal system aims to achieve $100 \%$ sensitivity (i.e., identify all errors) and $100 \%$ specificity (i.e., not induce any false alarms). For any system, there is a trade-off between sensitivity and specificity since the number of false alarms is increased if the sensitivity is increased by lowering the threshold for error detection.

An inherent problem of current IVD systems is poor sensitivity and specificity. A typical example of standard rectal IVD performed during intracavitary BT for cervical cancer is shown in Fig. 1. Figure 1 shows the measured accumulated diode dose per BT fraction compared with the calculated dose from the treatment planning system based on MR images. The discrepancy between measured and calculated doses was $-9 \% \pm 18 \%$ [mean and standard deviation (SD)]. There is a significant variation in the difference between calculated and measured doses, and discrepancies larger than $30 \%$ occurred in $10 \%$ of the treatment fractions. Numerous reports show examples of the same magnitude of deviations in clinical measurements, ${ }^{40,55-57}$ and notably such a measurement approach is likely to detect only gross errors $>50 \%$.

In order to avoid false alarms, current error decision criteria are usually high, which means that the error detection sensitivity is low. Currently, flat error criteria are mainly used in clinical practice. However, a flat criterion of, e.g., $10 \%$ may result in false alarms when the detector is close to the source, where positional uncertainties of the detector lead to dose uncertainties larger than $10 \% .{ }^{36}$ On the other hand, $10 \%$ may be too large for treatment error detection at larger sourcedetector distances where the influence of positional uncertainties is less. ${ }^{36}$

\section{DETECTORS: STATE OF THE ART}

Many dose-measurement systems have been used in BT in spite of the practical challenges encountered or the

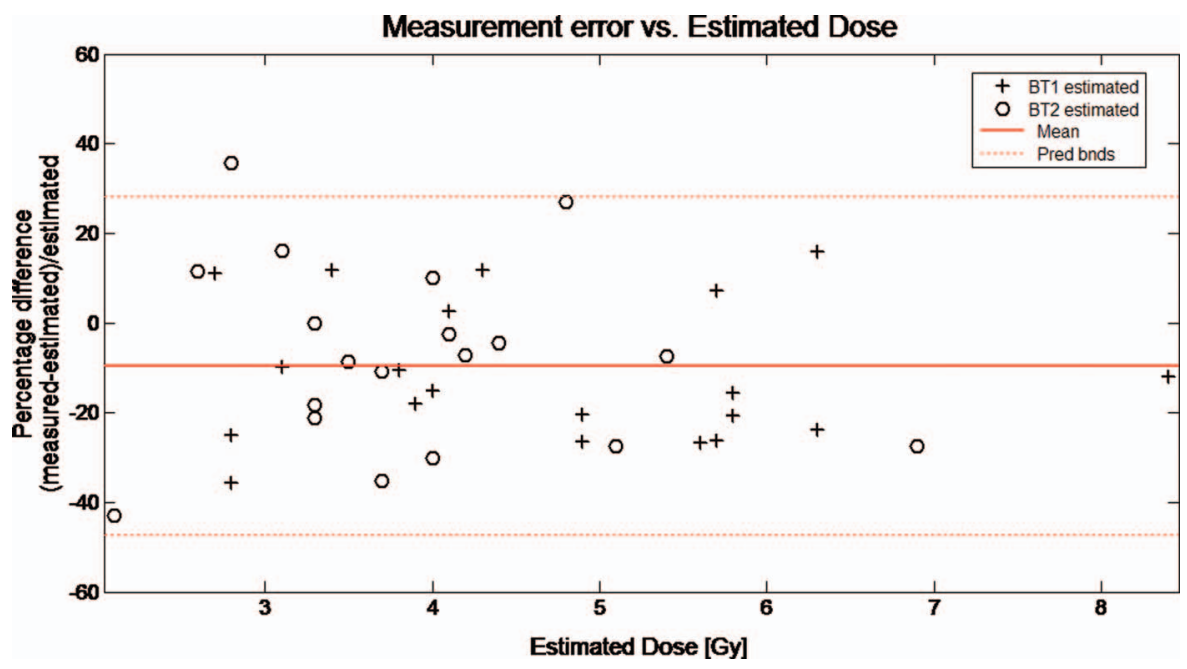

FIG. 1. Rectal IVD in PDR ${ }^{192}$ Ir cervix cancer BT with tandem ring applicator for BT fractions 1 (BT1) and 2 (BT2). Dashed lines indicate bounds of the $95 \%$ prediction interval. 
TABLE III. Characteristics of detectors and dosimetry systems of importance for precise routine IVD in brachytherapy. The items are rated according to: advantageous $(++)$, good $(+)$, and inconvenient $(-)$.

\begin{tabular}{|c|c|c|c|c|c|c|}
\hline & TLD & Diode & MOSFET & Alanine & RL & PSD \\
\hline Size & + & $+1-$ & $+/++$ & - & ++ & ++ \\
\hline Sensitivity & + & ++ & + & - & ++ & $+/++$ \\
\hline Energy dependence & + & - & - & + & - & ++ \\
\hline Angular dependence & ++ & - & + & + & ++ & ++ \\
\hline Dynamic range & ++ & ++ & + & - & ++ & ++ \\
\hline $\begin{array}{l}\text { Calibration } \\
\text { procedures, QA, } \\
\text { stability, robustness, } \\
\text { size of system, ease } \\
\text { of operation }\end{array}$ & + & ++ & ++ & - & $-1+$ & $+/++$ \\
\hline $\begin{array}{l}\text { Commercial } \\
\text { availability }\end{array}$ & ++ & ++ & ++ & ++ & - & + \\
\hline Online dosimetry & - & ++ & + & - & ++ & ++ \\
\hline Main advantages & $\begin{array}{l}\text { No cables, well } \\
\text { studied system }\end{array}$ & $\begin{array}{l}\text { Commercial } \\
\text { systems at } \\
\text { reasonable price, } \\
\text { well studied system }\end{array}$ & $\begin{array}{l}\text { Small size, } \\
\text { commercial system } \\
\text { at reasonable price }\end{array}$ & $\begin{array}{l}\text { Limited energy } \\
\text { dependence, no } \\
\text { cables }\end{array}$ & $\begin{array}{l}\text { Small size, high } \\
\text { sensitivity }\end{array}$ & $\begin{array}{l}\text { Small size, no } \\
\text { angular and energy } \\
\text { dependence, } \\
\text { sensitivity }\end{array}$ \\
\hline Main disadvantages & $\begin{array}{l}\text { Tedious procedures } \\
\text { for calibration and } \\
\text { readout, not online } \\
\text { dosimetry }\end{array}$ & $\begin{array}{l}\text { Angular and energy } \\
\text { dependence }\end{array}$ & $\begin{array}{l}\text { Limited life of } \\
\text { detectors, energy } \\
\text { dependence }\end{array}$ & $\begin{array}{l}\text { Not sensitive to low } \\
\text { doses, tedious } \\
\text { procedures for } \\
\text { calibration and } \\
\text { readout, not online } \\
\text { dosimetry, } \\
\text { expensive readout } \\
\text { equipment not } \\
\text { available in clinics }\end{array}$ & $\begin{array}{l}\text { Needs frequent } \\
\text { recalibration, stem } \\
\text { effect, not } \\
\text { commercially } \\
\text { available }\end{array}$ & Stem effect \\
\hline
\end{tabular}

shortcomings in their physical properties such as energy response, signal noise ratio, signal stability, readout precision, and positional accuracy. ${ }^{54}$ In principle, detectors that are commonly used for the EBRT absorbed dose measurements may be good candidates for application for IVD for BT. Few studies have been conducted in the past to compare the performance of these detectors for both high dose BT and EBRT. ${ }^{58-60}$ Thermoluminescent dosimeters (TLD), ${ }^{32,42,61-63}$ semiconductor diodes, ${ }^{30,64}$ metal-oxide-semiconductor field-effect transistors (MOSFET), ${ }^{33,34,65}$ radioluminescence (RL) and optically stimulated luminescence (OSL) detectors, ${ }^{66}$ plastic scintillation detectors (PSD), ${ }^{41,67-70}$ alanine detectors, ${ }^{39,71}$ radiophotoluminescent glass dosimeters (RPLGD) (Refs. 55, 56, and 72) have been used for IVD in BT to measure doses over an assortment of disease sites. The characteristics and the operation of these detectors are briefly described below, and have also been presented and discussed in the accompanying Vision 20/20 paper for EBRT, ${ }^{11}$ in review papers, ${ }^{73}$ as well as in textbooks. ${ }^{74,75}$

There is no ideal detector for all BT measurements because of the severe requirements needed and demands expected of the dosimeters. All currently used detectors exhibit varying degrees of artifacts such as volume-averaging, selfattenuation, response angular anisotropy, energy dependence, and a nonlinear dose response. Therefore, BT detector selection is crucial and is dictated by the radioactive source in question, the spatial locations of the measurement points with respect to the source, practical insertion(s) of the detectors, and the disease site. Only detector designs that can be easily inserted within either intraluminal catheters or special applicators inserted into patients should be considered. Table III presents an overview of characteristics of the most relevant detectors for IVD in BT and an evaluation of their advantages and disadvantages.

\section{V.A. TLDs and RPLGDs}

TLDs have been used for HDR ${ }^{192}$ Ir BT to measure the dose to the urethra and/or rectum during prostate treatments (24-28). TLDs come in a variety of materials and forms and can be shaped to easily fit into various areas of interest. The lithium fluoride (LiF) TLD rods are the most widely used form, because they can be easily inserted into catheters. However, they require special preparation (annealing, individual calibration, careful handling, fading correction, etc.) and do not provide a direct reading right after the treatment. The combination of these properties places them into a secondary choice compared to other dosimetry systems that are capable of providing reading in real time.

RPLGDs have a similar reading process to TLDs, except ultraviolet light (and not heat) is used to stimulate the detector. Irradiation of the silver-activated phosphate glass converts silver ions to stable luminescent centers; when exposed to ultraviolet light, the luminescent centers produce fluorescence in proportion to the absorbed radiation dose. These detectors 
have been used for IVD (42-44) by Nose et al. ${ }^{55,56}$ for HDR interstitial BT for head-and-neck cancer (61 patients) and pelvic malignancies (66 patients). In the case of the pelvic patients, the RPLGDs were sutured to the anterior wall of the rectum or positioned inside a Foley catheter inside the urethra. Deviations greater than $20 \%$ were found between the measured and calculated doses and were attributed to the independent movement of the organs and applicators between the planning and treatment delivery times.

\section{V.B. Semiconductor diodes}

Diodes are another type of semiconductor solid-state dosimeters, typically based on silicon. They are mostly used for EBRT but have been also employed for in vivo verification of BT plans. They have the advantage of providing users with an immediate readout, high sensitivity, good mechanical stability, fairly small size. Unfortunately, they are also known for the undesirable dosimetric characteristics which include directional dependence, energy dependence, temperature dependence, and changes in sensitivity due to radiation damage. As these characteristics affect the accuracy of the dosimetry system, they must be thoroughly characterized in phantom before clinical use for the specific radiation source or application. ${ }^{57,76}$

Waldhäusl et al. ${ }^{64}$ and Seymour et al. ${ }^{57}$ both used flexible diode array manufactured by PTW for IVD for patients undergoing HDR ${ }^{192}$ Ir treatments. Waldhäusl et al. ${ }^{64}$ evaluated suitability of rectal probes consisting of five diodes (separated by $15 \mathrm{~mm}$ ) and bladder probes consisting of a single diode for IVD of cervix cancer patients. Their measurements resulted in differences between calculated and measured doses ranging from $-31 \%$ to $+90 \%$ (mean $11 \%$ ) for the rectum and from $-27 \%$ to $+26 \%$ (mean $4 \%$ ) for the bladder. They also reported that shifts in probe position of $2.5 \mathrm{~mm}$ for the rectal probe and $3.5 \mathrm{~mm}$ for the bladder probe caused dose differences exceeding $10 \%$. Seymour et al. ${ }^{57}$ performed a dosimetric evaluation of the five diode array in the routine verification of planned dose inside the rectum for prostate (HDR) BT using a real-time planning system. The deviations between the diode measurements and the TPS predicted values ranged from $-42 \%$ to $+35 \%$ (with $71 \%$ of the measurements within $10 \%$ of the predicted values). Waldhäusl et al. ${ }^{64}$ and Seymour et al. ${ }^{57}$ estimated that the overall uncertainty involved in phantom dose measurement, after correcting for all the factors affecting diode response were $\pm 7 \%$ (SD) and $\pm 10 \%$ (SD), respectively.

\section{V.C. MOSFET detectors}

MOSFETs are miniature n- or p-type silicon ( $\mathrm{Si}$ ) semiconductors. For dosimetry, mostly p-type detectors are used. In general, MOSFETs as all semiconductors, exhibit temperature dependence. However, this effect has been overcome by specially designed dual-MOSFET-dual bias detectors ${ }^{77}$ produced by Best Medical Canada. These detectors can be produced either in standard size, or as micro-MOSFETs. The latter ones are particularly suitable for BT due to their small size $\left(8 \times 2.5 \times 1.3\right.$ and $0.2 \times 0.2 \times 5 \times 10^{-4} \mathrm{~mm}^{3}$ for external and sensitive volume dimensions, respectively). They are also available in the form of an array of five detectors. Micro-MOSFETs have almost isotropic response to radiation. Unlike single dual-MOSFET-dual bias detectors, MOSFET arrays have some temperature dependence (the temperature coefficient $\left.=0.6 \% /{ }^{\circ} \mathrm{C}\right) .{ }^{34}$ Most published studies on IVD in BT involve detectors and dosimetry system from Best Medical Canada. Another MOSFET dosimetry system used in BT comes from the Centre for Medical Radiation Physics (CMRP), University of Wollongong. ${ }^{78}$ It was found that their response degrades with exposure to radiation and varies with distance from a source due to its dose rate and energy dependence. MOSFET system from CMRP is not yet available in the market.

The two main disadvantages of MOSFETs for BT measurements are: (1) they are not tissue equivalent, which makes their dose response vary with radiation quality and (2) they have a rather limited lifetime. One of their main practicalities lies in their small size allowing their insertion inside intraluminal catheters or even needles and the possibility of having multiple MOSFET sensors along one single line. Recent coupling of a MOSFET detector with a sensor capable of detecting the position of the MOSFET in 3D (RADPOS), has renewed interest in their use.

MOSFETs have been used to characterize the HDR ${ }^{192} \mathrm{Ir}$ Leipzig surface applicators and found to agree with films and Monte Carlo calculations within 3\%. ${ }^{79}$

Cygler et ll $^{33}$ performed in 2006 a feasibility study using micro-MOSFETS (external dimensions: $1 \mathrm{~mm}$ diameter by $0.5 \mathrm{~mm}$ thickness) during permanent low-dose rate prostate implants as a tool for in vivo measurement of the initial dose rate within the urethra and as a mean to help evaluate the overall quality of the implant. In this study, IVD in the urethra was carried out by moving the MOSFET detector along the urethra in $1 \mathrm{~cm}$ steps. The estimated uncertainty of the MOSFET position was within $\pm 1 \mathrm{~mm}$. The detector was left at each position to accumulate dose for $10 \mathrm{~min}$, long enough to provide a reasonable measurement signal.

Bloemen-van Gurp et al. ${ }^{34,80}$ performed two studies in 2009 using a linear MOSFET array during permanent prostate implant. The overall uncertainty in the measurement procedure, determined in a simulation experiment, was $8.0 \%$ and an action level of $\pm 16 \%$ ( $2 \mathrm{SD}$ ) for detection of errors in the implantation procedure is achievable after validation of the detector system and measurement conditions. In their second study, they reported on a technique to monitor the dose rate in the urethra and performed IVD in five patients during and after implantation of ${ }^{125}$ I seeds. They concluded that IVD with MOSFET array during the procedure is a feasible technique to evaluate the dose in the urethra for permanent prostate BT. This study showed the challenges of performing IVD in BT and the extensive analysis needed to reach a meaningful conclusion.

Cherpak et al. ${ }^{81}$ performed real-time measurements of urethral dose and position using a RADPOS Array (Best Medical Canada) during permanent seed implantation for prostate BT. RADPOS Array is a modified RADPOS detector. ${ }^{82}$ 
Cherpak et al. ${ }^{81}$ performed measurements on 17 patients and concluded that the modified RADPOS detector with MOSFET array instead of a single MOSFET is able to provide accurate real-time dose information which can be used to monitor dose rates while implantation is performed and to estimate total integrated dose. Reniers et al. ${ }^{83}$ in 2012, in a feasibility study on a phantom, have studied the utility of using a RADPOS system for IVD for gynecological BT. They reported that the RADPOS sensor, if placed, for example, in the bladder Foley balloon, would detect a $2 \mathrm{~mm}$ motion of the bladder, at a $5 \%$ chance of a false positive, with an action level limit of $9 \%$ of the dose delivered. The authors also found that source position errors caused by, e.g., a wrong first dwell position, are more difficult to detect. With a single RADPOS detector positioned in the bladder, dwell position errors below $5 \mathrm{~mm}$ and resulting in a dose error within $10 \%$ could be detected in the tandem but not in the colpostats.

\section{V.D. Alanine}

$\mathrm{L}-\alpha$-alanine is a nonessential amino acid that has been used for electron paramagnetic resonance (EPR) dosimetry in radiation therapy. Alanine can exist in a form of a white powder or can be formed in the shape of rods, pellets, films, etc. ${ }^{84}$ During irradiation of alanine, permanent free radicals are formed. The concentration of these radicals is proportional to absorbed dose and can be measured by EPR spectroscopy. So alanine is a chemical dosimeter, since dose measurement is based on detection of chemical species formed during irradiation. Alanine has some important advantages as a dosimeter. Its response is almost independent of radiation energy and dose rate. It can be formed into small shapes and requires no bias or wiring. The readout of the radical concentration is nondestructive and has very little fading with time.

Unfortunately, it also has disadvantages that limit its usefulness in clinical dosimetry. The main disadvantage is a requirement of expensive EPR equipment not easily available in clinics. Alanine does not provide an immediate readout of the dose and it is generally insensitive to doses $<2$ Gy. It also has some temperature and humidity dependence. In spite of these limitations, some feasibility studies were conducted to evaluate its usefulness in BT. Apart from the phantom studies aimed mostly at the characterization of the radioactive sources, ${ }^{85-87}$ few reports exist on using alanine for IVD of gynecological treatments. ${ }^{39,88}$ Alanine is commercially available, but the tedious readout procedure needs to be established individually in each department. At present, use of alanine in the clinics is very limited due to the readout equipment being very expensive and it is hard to foresee that this will change in the near future.

\section{V.E. RL and OSL detectors}

Recent advances in optically stimulated luminescence dosimeters (OSLDs) have opened new possibilities for their application in radiation therapy.${ }^{89,90}$ OSLDs are gradually replacing TLDs as personal radiation monitoring dosimeters for industry and public health workers because of their instanta- neous reading capabilities, faster processing, and the ability to store their reading without much degradation of the optical signal (permanent record). To operate in the OSL mode, the crystals need an external stimulation (laser or diode) to extract the reading postirradiation. The same crystal can also operate in the RL mode providing the information about the dose rate during the irradiation, thus, serving as a true real-time online detector. $\mathrm{Al}_{2} \mathrm{O}_{3}: \mathrm{C}$ can be formed as crystals with diameter $<1 \mathrm{~mm}$ which can fit inside BT catheters. Even though the sensitive detecting material of these detectors $\left(\mathrm{Al}_{2} \mathrm{O}_{3}: \mathrm{C}\right)$ is not water-equivalent, they have good potential for IVD in BT due to their ability to provide feedback in real time.

RL systems for online dose measurements are not available. However, a prototype system developed by Andersen et $a{ }^{66}{ }^{6}$ in 2009 was investigated to facilitate the prevention and identification of dose delivery errors in remote afterloading BT. The system consists of a small $\mathrm{Al}_{2} \mathrm{O}_{3}$ : C crystal coupled with the optical fiber which carries the light signal generated in the crystal to the detection system. The system is capable to operate either in the RL or OSL mode. The system allows for automatic online IVD directly in the tumor region using small detector probes that fit into applicators such as standard needles or catheters. In the RL mode, the system is capable of measuring the absorbed dose rate (with a $0.1 \mathrm{~s}$ time resolution) and in the OSL mode-the total absorbed dose. The calibrated system was found to be linear in the tested dose range. The reproducibility for the RL/OSL measurements was found to be $1.3 \%$ (SD). Under certain conditions, the RL signal could be greatly disturbed by the socalled stem signal (i.e., unwanted light generated in the fiber cable upon irradiation). ${ }^{91}$ When taking into account the effect of reproducibility, energy response, angular dependence, stem effect, instrument and crystal temperature, light guide transmission on the reading, and the calibration uncertainty, the combined estimated uncertainty budget was found to be $8 \%$ (SD) and 5\% (SD) for RL and OSL, respectively. This initial study was followed up with a clinical protocol study on five cervix cancer patients undergoing PDR BT. ${ }^{35}$ For three of the patients, the authors found no significant differences $(p>0.01)$ between IVD and TPS calculated reference values (TG 43) at the level of dose per dwell position, dose per applicator, or total dose per pulse. For the two other patients, they noted significant deviations for three individual pulses and for one dosimeter probe. These differences could have been due to applicator movement during the treatment and/or an incorrectly positioned probe, respectively.

Kertzscher et ll $^{36}$ in 2011 tested the ability of a RL system to identify afterloading PDR and HDR gynecological and prostate BT errors in real time using a novel statistical error decision criterion. Their phantom studies showed that out of 20 interchanged guide tube errors, time-resolved analysis identified 17 while accumulated dose identified only two. Accumulated dose comparisons could leave $10 \mathrm{~mm}$ dosimeter displacement errors unidentified and while dose rate comparisons correctly identified displacements $\geq 5 \mathrm{~mm}$. This phantom study demonstrated that $\mathrm{Al}_{2} \mathrm{O}_{3}: \mathrm{C}$ real-time dosimetry has the potential to detect interchanged guide tube errors during PDR and HDR BT and has the ability to identify applicator 
displacements $\geq 5 \mathrm{~mm}$. Another important aspect of this study was to highlight the shortcoming of using a constant error criterion and instead to recommend using a statistical error criterion.

\section{V.F. Plastic scintillation detectors}

Plastic scintillation detectors are a promising option for IVD. PSDs are composed of organic scintillation material (scintillator or scintillating optical fiber) that emits light proportionally to the dose deposited in their detector volume. The light produced in the detector is usually optically coupled to a fiber-optic light guide and transmitted toward a photodetector. The properties of plastic scintillation detectors have been studied for high-energy external beams. ${ }^{67,92}$ High spatial resolution, linearity with dose, energy independence in the megavoltage energy range, and water equivalence are among the advantages that have been demonstrated for such detectors. Cerenkov light production has also been identified in the optical guide. ${ }^{67,92,93}$ This light component, often referred to as stem effect, is produced in the fiber when struck by radiation over a certain energy threshold, which depends on fiber material, and needs to be removed to perform accurate dosimetry. Different methods have been proposed to efficiently account for this spurious effect. ${ }^{94,95}$ Using these detectors, possibility of real-time IVD has been demonstrated under external beam radiation ${ }^{96}$ and accuracy of better than $1 \%$ has been achieved. New evidence pointing to possible temperature dependence has recently been reported as a characteristic being exhibited by plastic scintillation detectors. ${ }^{97}$ An average of a $0.6 \%$ decrease in measured dose $/{ }^{\circ} \mathrm{C}$ relative to dose measured at room temperature was observed in the range of $15-50{ }^{\circ} \mathrm{C}$. At the present time, it is not clear if this thermal dependence comes from the construction of the materials used in the design, the composition of the organic scintillator itself, or is coming from the optical train. Since the exact mechanism is not well understood, this early communication concluded that further research is warranted.

PSDs have the capability to perform accurate online IVD during HDR ${ }^{192}$ Ir BT, ${ }^{58,68,69}$ because they could be cut and shaped in size that can be easily inserted into catheters or arranged around applicators that are commonly used in BT. Lambert et $a l .{ }^{58}$ performed a comparative phantom study of PSDs to other commercially available detectors (MOSFET, diamond detector, and TLD). Based on size, accuracy, and real-time possibilities, the authors claimed that PSDs showed the best combination of characteristics to perform dosimetry during HDR ${ }^{192}$ Ir BT. Because the energy emission spectrum of an ${ }^{192}$ Ir radiation source is mainly higher than the Cerenkov production threshold energy, the need for a stem removal technique has been stressed for clinically relevant situations. ${ }^{68} \mathrm{~A}$ study using a prostate phantom was performed by TherriaultProulx et al. ${ }^{69}$ The authors used a red-green-blue (RGB) photodiode as the photodetection component, allowing for the removal of the Cerenkov component through the polychromatic approach. Thirteen catheters were implanted in the phantom: 12 for dose delivery and one for the PSD. The PSD dose rates were measured in real-time during the treatment delivery us- ing a HDR ${ }^{192}$ Ir source. The average accumulated dose measured at the PSD location for five consecutive treatment deliveries was $275 \pm 14 \mathrm{cGy}$, in good agreement with the expected dose of 275 cGy as calculated by the treatment planning system. Another phantom study using an array of 16 PSDs in an insertable applicator that enables quality assurance of the treatment delivery and provides an alert to potential radiation accidents during HDR BT treatments has been performed by Cartwright et al. ${ }^{70}$ The system presented is capable of measuring doses in a phantom for $1 \mathrm{~s}$ exposures with an uncertainty between $2 \%$ and $3 \%$ for most of the PSDs.

Suchowerska et al ${ }^{41}$ described a plastic scintillator system consisting of a $0.5 \mathrm{~mm}$ diameter by $4 \mathrm{~mm}$ length BC400 scintillator optically coupled to a $0.48 \mathrm{~mm}$ core PMMA optical fiber to transmit the signal. They have used a PTW-Freiburg OPTIDOS Reader to read the signal produced by their scintillator detector. ${ }^{41}$ The OPTIDOS reader, consisting of a photomultiplier and an electrometer unit, was originally designed to measure the dose rate of ${ }^{90} \mathrm{Sr} /{ }^{90} \mathrm{Y}$ beta emitting sources as well as for ${ }^{32} \mathrm{P}$ intravascular BT using different plastic scintillator detectors designed and manufactured by PTW-Freiburg. Note that the PTW-Freiburg scintillation detectors and OPTIDOS reader system are no longer commercially available. The clinical feasibility of this study examined urethral dose measurements in 24 patients during HDR prostate BT. The authors of this study did not report any temperature dependence for their measurement performed inside the urethra nor any stem effect when using these PSDs. After the first 14 patients, improvements to the dosimeter system design were implemented that increased positional accuracy and system reliability, with the maximum dose departure from the calculated dose being within $9 \%$ for all of the ten remaining patients and concluded that dose to the urethra could be accurately measured using the improved system.

At the present time there are no PSDs or PSD systems that are commercially available for use for in vivo dosimetry for BT. The only PSD system available commercially is the EXRADIN W1 Scintillator manufactured by Standard Imaging which was designed for external beam radiotherapy and more specifically for the characterization of small field dosimetry (http://www.standardimaging.com/product_ home.php?id=124).

\section{DIRECTIONS FOR THE FUTURE}

\section{VI.A. Detectors and technology}

Most of the development efforts for IVD systems have been focused to the front end of the detection system: the detector element(s) or the sensitive material and their dosimetric characteristics to correct for the effects influencing their response. Future developments would have to be focused on the back end of the detection system utilized to process the data (i.e., the readings of the detector) to make the next generation "IVD systems" user friendly with automatic data display, requiring less manpower, with no postdata analysis or special expertise needed. Furthermore, in order to be successful for widespread use, any new technological innovation for such 
systems will have to be well integrated into the routine clinical application being targeted.

Given existing alternatives for accurate BT dosimeters, future requirements will likely be made on IVD systems that can monitor the dose in real time, are sensitive to displacements of all applicators involved in the treatment, can identify organ motion, and that can provide immediate alerts of any potential gross error during ongoing treatments. Passive detectors, such as TLDs, OSLDs (when used in the passive mode), RPLDs, alanine, radiographic and radiochromic films, gels, are not capable of monitoring the time-resolved treatment delivery. A few studies have introduced the possibility of using the instantaneous dose rate for patient QC checks. ${ }^{31,35,36}$ These studies indicate that time-resolved dosimetry has potential to be more sensitive to error detection than dose verification based on integral dose. Therefore, future research will probably focus on further developing systems that would have the ability to sample the dose rate in real time while the radiation treatment is being delivered to the patient. Currently, good candidates for real-time IVD are RL, ${ }^{35,36}$ PSDs, ${ }^{69}$ and MOSFETs. These detectors are capable of sampling or measuring the dose rate which could be useful for certain applications for which thresholds in dose deviations could be set and/or upper and lower limits for dose values to prevent gross errors and potentially alert the users whether the treatment should or should not be terminated. Although semiconductor diodes provide an immediate readout, they are not good candidates for real-time IVD due to their many undesirable dosimetric characteristics (see Table I).

Recently, it has been shown that it is possible to develop detectors composed of multiple different color PSDs (mPSD) using a single optical transmission line and capable of measuring the dose accurately (Fig. 2). ${ }^{98}$ Detectors of this type could be used for LDR or HDR interstitial BT applications. Additional "carrier catheters" could be sutured within the vicinity of the implant or nearby a critical structure to monitor the dose from the planned implant. The "carrier catheters" are not part of the implant but additional intraluminal catheters for the sole purpose to accommodate the detectors. The readings consist of the light spectrum emitted by each color PSD. The extraction of each corresponding color from the total spectrum measured will provide the dose deposited at corresponding color PSD. This type of detector has the advantage of simultaneous dose rate measurements in multiple points with the potential to improve error detection capabilities.

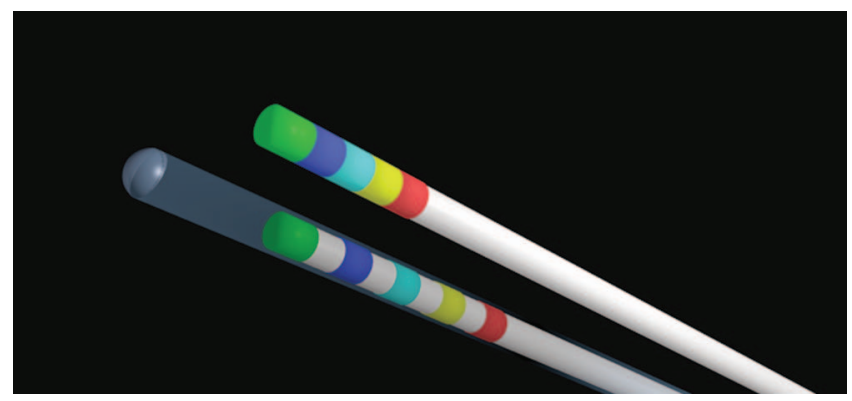

FIG. 2. mPSD line detector inserted into a flexible catheter.

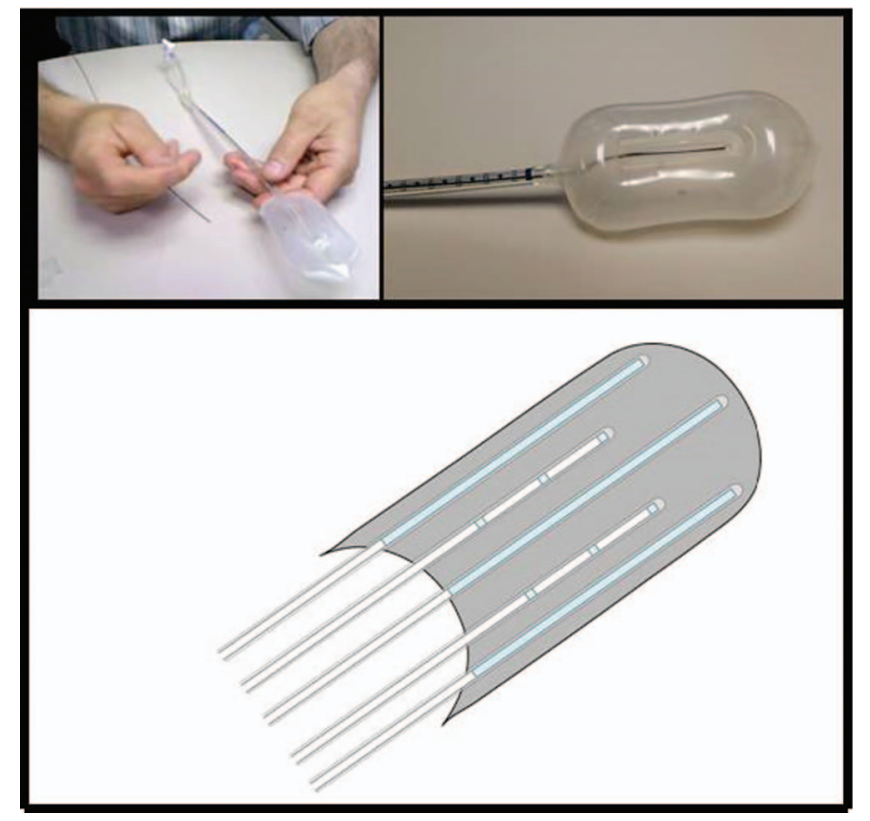

FIG. 3. An example of integrating a detector to a rectal balloon. In this case, a $0.5 \mathrm{~mm}$ diameter by $1.0 \mathrm{~mm}$ long plastic scintillating fiber coupled to a clear transmitting fiber-optic and an inflatable rectal balloon (upper left) were fully integrated into an inflatable rectal balloon with the detector permanently moulded to it (upper right). Another example illustrating integrating a combination of line detectors and point detectors integrated to an applicator is shown below. A semicylindrical cut of the rectal balloon surface onto which, in this case scintillating line and point probes are attached for detection of dose along the rectal wall. The individual line and point probes slide into capillary tubes permanently attached to the balloon surface.

Stable and clinically relevant detector positioning remains one of the most important and critical issues for IVD. Insertion of additional detector catheters may be performed with the purpose of optimizing detector positioning, e.g., by inserting an additional interstitial needle for housing a dosimeter probe in the tumor region. A smaller invasive impact may be achieved by inserting a sterilized dosimeter in the urethral catheter for urethral dose measurements. However, such invasive procedures raise ethical issues as discussed in Sec. IV.A. Another option is to mould detectors within an applicator (see example in Fig. 3), which has advantages such as fixing the sensitive volume within the geometry of the applicator and therefore reducing the positional uncertainty of the detector. The applicator could potentially be designed to constrain movements of OARs and/or the target region. Similar measures could be achieved with a urethral catheter with a closed-end lumen dedicated for housing a dosimeter probe in the urethra or along the bladder wall.

Improved integration between afterloader and dosimetry system is warranted. The Bebig Multisource afterloader (by Eckert \& Ziegler Bebig GmbH, Berlin, Germany) offers integrated diode IVD where the dosimetry system can be operated from the control unit. It permits the specification of dose limits and the display of rectum and bladder doses. A further step forward for integration would be automated DICOM import to the dosimeter software and signal communication between the dosimeter software and the afterloader. 
The communication would help synchronize the dosimeter readout with the onset of the treatment, hence, optimize the comparison between the delivered treatment with the time and source coordinates from the imported DICOM RT plan. Another attractive solution for future IVD in BT is the integration of one or several dosimeter probes into the afterloading machinery. Such an afterloader could offer motorized feeding of dosimeter probes into BT catheters already inserted into the patient but unoccupied by the source. Positioning technology attached onto both the source wire and dosimeter probe could both minimize their positional uncertainties and optimize the relative source-to-dosimeter distances. In vivo detector positioning directly inside the source catheters, e.g., needles and intracavitary tubes, would in principle already be possible with sufficiently small dosimeter probes as the RADPOS, MOSFETs and fiber-coupled scintillator, and RL probes.

Recently developed RADPOS system ${ }^{82,99,100}$ allows for simultaneous measurement of dose and position. This detector is particularly suited for BT applications and is being explored to study not only its sensitivity for error detection during HDR treatments ${ }^{81}$ but also for applicator movement during vaginal cylinder treatments and prostate movement during permanent prostate implants. ${ }^{81}$ RADPOS is capable of detecting prostate movement during each needle insertion, as well as the change in the urethral dose after the removal of the ultrasound probe from the rectum. ${ }^{81}$ Since the RADPOS probe is visible on $\mathrm{x}$ ray images, it allows to link the coordinates of the point of measurement before and during the treatment.

\section{VI.B. Error detection criteria}

A significant challenge of future IVD is to define relevant error/decision criteria which take into account that both sensitivity and specificity of treatment error detection is sound and robust in a clinical setting. More advanced error decision criteria could involve a statistical approach where the comparison between calculated and measured doses takes into account the uncertainty components for both calculated and measured doses. The calculated dose is based on assessment of both source and detector positions as they are identified, e.g., in 3D images (US, CT, or MRI) during treatment planning. The identification of both source and detector position is correlated with specific reconstruction uncertainties. The influence of positional uncertainties on the calculated dose may be quantified by simulation which incorporates the source/detector geometry as known from the TPS and an assumption of a certain distribution of positioning errors. ${ }^{35,36}$ Furthermore, dose calculation is correlated with uncertainties due to the shortcomings of dose calculation algorithms imposed, e.g., by inhomogeneities and scatter conditions. The uncertainty on the measured dose has a number of components which are related to the detector characteristics. Based on comparison between calculated and measured doses, the measurement should be categorized into "Alarm" or "No alarm." A certain action level should be defined by balancing between sensitivity and false alarms (Table II). The action level can be based on a statistical evaluation of the incidence of a type 1 error (false alarm), e.g., $p=0.05$ or $p=0.01$ which means that $5 \%$ or $1 \%$ of all correct treatments will induce a false alarm, respectively.

The accuracy of the "Alarm classification" depends on the magnitude of components in the entire uncertainty budget and as such can be optimized by reducing uncertainties. These factors relate to the dosimeter technology, e.g., calibration accuracy and energy dependence, and to clinical procedures, e.g., image reconstruction and patient-DICOM matching. An optimized dosimetry integration into the entire BT workflow would reduce the incidence of false alarms (type I errors) and/or decrease the failure to detect treatment errors (type II errors). This would improve treatment error detection and minimize the risk of making erroneous decisions regarding unnecessary treatment interruptions or termination.

An interesting strategy for further improvement of error decision criteria could be development of a gamma analysis for IVD according to the principles which have been used in EBRT for comparison of measured and expected doses, e.g., with EPID dosimetry. ${ }^{101}$ With a gamma analysis an error criterion would be based not only on a dose difference but could also combine it with a distance-to-agreement criterion. In such an analysis, the upfront knowledge of the geometrical uncertainties of the detector and source positioning could be incorporated into the model in order to decide on relevant distance-to-agreement criteria.

\section{VI.C. Clinical operation, infrastructure, and workflow}

Patient involvement should be considered so that the patient is as comfortable as possible and not stressed by additional procedures if possible. Current rectal diode systems and/or additional catheter insertion into the urethra/bladder involve some patient discomfort. Reduction of detector size may contribute to improved patient acceptance. Optimally, the use of BT source catheters for in vivo detectors, as discussed in Sec. VI.A, would not require any extra insertion of catheters, and could potentially make the in vivo system completely "invisible" for the patient.

A major barrier for routine implementation of IVD is the added workload for the personnel. Developments of new systems should involve considerations of time consumption, clinical robustness, and ease of use. The Quality Management Program should be optimized in order to limit the time consumption for the personnel. The measurements should be presented and visualized to the user in such a way that the evaluation and interpretation of the results is as straightforward as possible and so that a clear action can be taken from the measurements. The IVD system should also not distract personnel from other important tasks related to treatment planning and dose delivery.

An example of a general dosimetry integration into an image guided BT workflow is shown in the right pane of Fig. 4. Some potential sources of errors generated during such treatment procedures are specified in the left pane, where those detectable with IVD are highlighted.

An optimal dosimetry integration would involve source applicators with integrated dosimeter housing, which would 


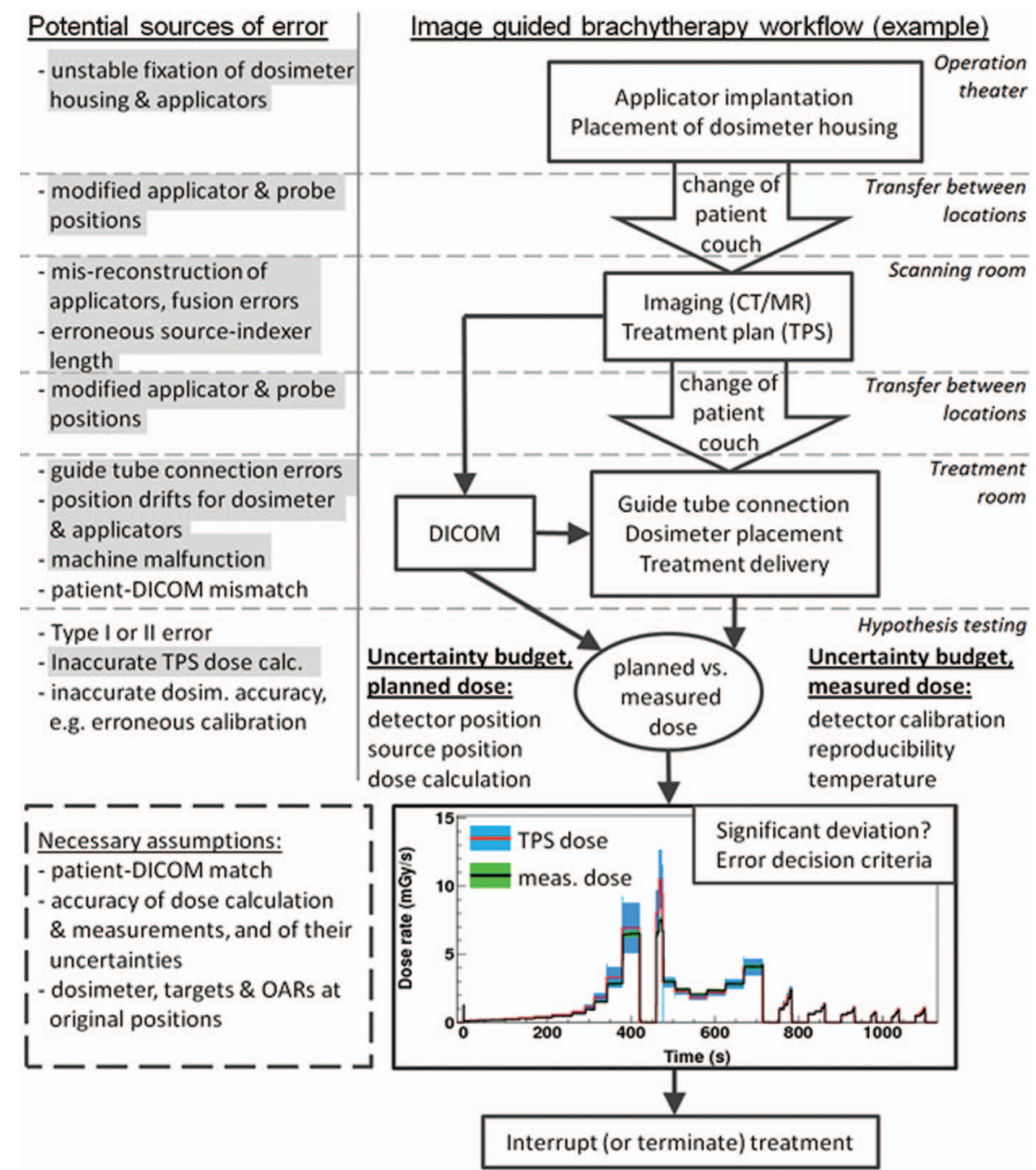

FIG. 4. Example of workflow for CT/MR image guided BT. Potential discrepancies between planned and delivered treatments may originate from errors (see left pane) presented at different stages of the treatment workflow (see right pane). In principle, in vivo dosimetry addresses detection of the highlighted error types.

not require additional work for the physician during the implantation stage in the operation theatre. As discussed in Sec. VI.A, the dosimeter housing could provide anatomical stability in order to minimize the impact of the patient transfer stages on the original positions of the source and dosimeter catheters. Furthermore, contrast material could be more systematically incorporated into the catheters in order to aid accurate reconstruction of the dosimeter and source dwell positions from CT/MR images. MRI imposes significant challenges since traditional metal markers do not induce contrast on MRI, hence, alternative fluid markers may be used for indication of reference points or source/dosimeter catheters. In addition to control checks by the staff, connection errors between the afterloader and the dosimeter, and their designated catheters, could be reduced, e.g., by using different connection mechanisms for the dosimeter and source catheters.

Improvements of sterilization options would also have potential to improve the clinical workflow. National or hospital requirements do not always match with company requirements, and may prevent the use of IVD. Therefore, larger flexibility of sterilization methods is needed for overall application of the IVD techniques. Introduction of single use sterile sheaths for the dosimeter probes may be a practical approach in certain clinical scenarios.

\section{VI.D. Specific key questions for future in vivo dosimetry}

There is significant potential for IVD to move forward into a new era of increased sensitivity and specificity for error detection. However, a number of essential issues need to be addressed as discussed above. The following is a list of important questions when considering new routes or approaches:

- Detector selection: Is the detector favorable for BT in terms of its characteristics, size, and sensitivity?

- Operation of in vivo system: Is the system practical in terms of calibration, quality management procedures, stability, robustness, size of the system, ease of operation? 
- Infrastructure: Is online dosimetry exploited? Is automatic analysis of data incorporated?

- Detector position: Is the positional accuracy of the detector addressed and optimized?

- Error decision criteria: Fixed level or statistical approach?

- Sensitivity and specificity: Is the sensitivity and specificity of the system evaluated?

- Communication: Is the dosimetry system able to communicate with TPS and/or afterloader?

- Prospective IVD: Is IVD used prospectively and systematically to evaluate the incidence of errors?

Recent progress of IVD shows that some of these points have already been addressed individually in different publications. However, the aim and vision for IVD is to create a system that embraces all the above aspects.

\section{CONCLUSIONS}

The specific role of IVD in BT needs to be defined and established. So far, recommendations for BT practice rarely include the recommendation to perform IVD. This fact is most likely mainly influenced by the poor ability of current systems to detect errors. Nevertheless, IVD is used in several departments and there is good potential to improve its utility and use. Most importantly, incorporation of routine IVD into clinical practice requires that the error detection capability is improved. Second, streamlined procedures are needed which are automatic as far as possible so that the work load is not excessive and so that the medical physicist is not distracted by technical operation. The systems need to be commercially available and affordable. Further utilization and dissemination of IVD will only be feasible if it is cost effective to assign resources for its infrastructure. Altogether, it should be demonstrated that IVD can be developed to become sensitive to errors that may have clinical impact and avoidance of errors should be verified.

Proceeding from this, it is crucial to come to a comprehensive description of errors in order to make progress within BT safety. Systematic IVD in a multicenter setting would be a step forward for detecting and understanding errors and their frequencies. Support of international databases for error reporting and for logging user experiences is important, since publications from academic centers do not reflect the majority of patient treatments. The Radiation Oncology Safety Information System (ROSIS) database supported by ESTRO already supports reporting of BT incidents and the first reports from ROSIS include such incidents. ${ }^{102}$ Such practice has potential to facilitate significant improvements in quality for BT by (1) advancing the use of IVD systems in order to prevent and avoid BT errors and (2) making it possible to focus on the most relevant sources of errors.

It is the opinion of the authors that IVD in BT is currently not well established in clinical routine to be used for sensitive and specific error detection. However, this situation should change over the coming years by investing in development of IVD with the goal of establishing an efficient means of inde- pendent verification of dose delivery in BT. As soon as IVD in BT becomes better established, there will be a strong need for recommendations from professional organizations (e.g., ESTRO, ASTRO, AAPM, ABS).

\section{ACKNOWLEDGMENT}

The authors have no financial ties to any of the discussed detector types and/or to a commercial company.

a) Author to whom correspondence should be addressed. Electronic mail: karitand@rm.dk

${ }^{1}$ International Commission on Radiation Units and Measurements, "Dose and volume specification for reporting intracavitary therapy in gynecology," ICRU Report No. 38 (ICRU, Bethesta, MD, 1985).

${ }^{2}$ R. Pötter et al., "Recommendations from gynaecological (GYN) GEC ESTRO working group (II): Concepts and terms in 3D image-based treatment planning in cervix cancer brachytherapy-3D dose volume parameters and aspects of 3D image-based anatomy, radiation physics, radiobiology," Radiother. Oncol. 78, 67-77 (2006).

${ }^{3}$ T. Major, C. Polgar, K. Lovey, and G. Frohlich, "Dosimetric characteristics of accelerated partial breast irradiation with CT image-based multicatheter interstitial brachytherapy: A single institution's experience," Brachytherapy 10, 421-426 (2011).

${ }^{4}$ W. S. Bice, Jr. et al., "Centralized multiinstitutional postimplant analysis for interstitial prostate brachytherapy," Int. J. Radiat. Oncol., Biol., Phys. 41, 921-927 (1998).

${ }^{5}$ M. J. Rivard, J. L. Venselaar, and L. Beaulieu, "The evolution of brachytherapy treatment planning," Med. Phys. 36, 2136-2153 (2009).

${ }^{6}$ J. Van Dyk, R. B. Barnett, J. E. Cygler, and P. C. Shragge, "Commissioning and quality assurance of treatment planning computers," Int. J. Radiat. Oncol., Biol., Phys. 26, 261-273 (1993).

${ }^{7}$ B. Mijnheer, "State of the art of in vivo dosimetry," Radiat. Prot. Dosim. 131, 117-122 (2008).

${ }^{8}$ AAPM, "Report of TG 62 of the Radiation Therapy Committee: Diode in vivo dosimetry for patients receiving external beam radiation therapy," AAPM Report No. 87 (Medical Physics Publishing, Madison, WI, 2005).

${ }^{9}$ A. J. Vinall, A. J. Williams, V. E. Currie, E. A. Van, and D. Huyskens, "Practical guidelines for routine intensity-modulated radiotherapy verification: Pre-treatment verification with portal dosimetry and treatment verification with in vivo dosimetry," Br. J. Radiol. 83, 949-957 (2010).

${ }^{10}$ A. Mans et al., "Catching errors with in vivo EPID dosimetry," Med. Phys. 37, 2638-2644 (2010).

${ }^{11}$ B. Mijnheer, A. S. Beddar, J. Izewska, and C. S. Reft, "In vivo dosimetry in external beam radiotherapy," Med. Phys. 40(7), 070903 (19pp.) (2013).

${ }^{12}$ P. O. Lopez, P. Andreo, J.-M. Cosset, A. Dutreix, and T. Landberg, "Prevention of accidental exposures to patients undergoing radiation therapy," ICRP Publication 86, Annals of the ICRP (Pergamon, New York, 2000).

${ }^{13}$ L. P. Ashton, J.-M. Cosset, V. Levin, A. Martinez, and S. Nag, "Prevention of high-dose-rate brachytherapy accidents," ICRP Publication 97, Annals of the ICRP (Pergamon, New York, 2004).

${ }^{14}$ IAEA, "Lessons learned from accidental exposures in radiotherapy," IAEA Safety Report Series 17 (IAEA, Vienna, 2000).

${ }^{15} \mathrm{~K}$. Yoshida et al., "Needle applicator displacement during high-dose-rate interstitial brachytherapy for prostate cancer," Brachytherapy 9, 36-41 (2010).

${ }^{16} \mathrm{~T}$. Simnor et al., "Justification for inter-fraction correction of catheter movement in fractionated high dose-rate brachytherapy treatment of prostate cancer," Radiother. Oncol. 93, 253-258 (2009).

${ }^{17}$ A. A. de Leeuw, M. A. Moerland, C. Nomden, R. H. Tersteeg, J. M. Roesink, and I. M. Jürgenliemk-Schulz, "Applicator reconstruction and applicator shifts in 3D MR-based PDR brachytherapy of cervical cancer," Radiother. Oncol. 93, 341-346 (2009).

${ }^{18} \mathrm{~N}$. Milickovic et al., "4D analysis of influence of patient movement and anatomy alteration on the quality of 3D U/S-based prostate HDR brachytherapy treatment delivery," Med. Phys. 38, 4982-4993 (2011).

${ }^{19}$ K. Koedooder, W. N. van, H. N. van der Grient, Y. R. van Herten, B. R. Pieters, and L. E. Blank, "Safety aspects of pulsed dose rate 
brachytherapy: Analysis of errors in 1300 treatment sessions," Int. J. Radiat. Oncol., Biol., Phys. 70, 953-960 (2008).

${ }^{20} \mathrm{~L}$. Beaulieu et al., "Report of the Task Group 186 on model-based dose calculation methods in brachytherapy beyond the TG- 43 formalism: Current status and recommendations for clinical implementation," Med. Phys. 39, 6208-6236 (2012).

${ }^{21}$ J. G. Sutherland, K. M. Furutani, Y. I. Garces, and R. M. Thomson, "Model-based dose calculations for ${ }^{125}$ I lung brachytherapy," Med. Phys. 39, 4365-4377 (2012).

${ }^{22} \mathrm{H}$. Afsharpour et al., "Consequences of dose heterogeneity on the biological efficiency of ${ }^{103}$ Pd permanent breast seed implants," Phys. Med. Biol. 57, 809-823 (2012).

${ }^{23}$ S. A. White, G. Landry, G. F. van, F. Verhaegen, and B. Reniers, "Influence of trace elements in human tissue in low-energy photon brachytherapy dosimetry," Phys. Med. Biol. 57, 3585-3596 (2012).

${ }^{24}$ ICRU, International Commission on Radiation Units and Measurements, "Dose and volume specification for reporting interstitial therapy," ICRU Report No. 58 (ICRU, Bethesta, 1997).

${ }^{25} \mathrm{~S}$. Nag et al., "The American Brachytherapy Society recommendations for low-dose-rate brachytherapy for carcinoma of the cervix," Int. J. Radiat. Oncol., Biol., Phys. 52, 33-48 (2002).

${ }^{26}$ A. N. Viswanathan and B. Thomadsen, "American Brachytherapy Society consensus guidelines for locally advanced carcinoma of the cervix. Part I: General principles," Brachytherapy 11, 33-46 (2012).

${ }^{27}$ B. J. Davis et al., "American Brachytherapy Society consensus guidelines for transrectal ultrasound-guided permanent prostate brachytherapy," Brachytherapy 11, 6-19 (2012).

${ }^{28}$ G. Kovacs et al., "GEC/ESTRO-EAU recommendations on temporary brachytherapy using stepping sources for localised prostate cancer," Radiother. Oncol. 74, 137-148 (2005).

${ }^{29} \mathrm{C}$. Salembier et al., "Tumour and target volumes in permanent prostate brachytherapy: A supplement to the ESTRO/EAU/EORTC recommendations on prostate brachytherapy," Radiother. Oncol. 83, 3-10 (2007).

${ }^{30} \mathrm{R}$. Alecu and M. Alecu, "In vivo rectal dose measurements with diodes to avoid misadministrations during intracavitary high dose rate brachytherapy for carcinoma of the cervix," Med. Phys. 26, 768-770 (1999).

${ }^{31}$ K. Tanderup, J. J. Christensen, J. Granfeldt, and J. C. Lindegaard, "Geometric stability of intracavitary pulsed dose rate brachytherapy monitored by in vivo rectal dosimetry," Radiother. Oncol. 79, 87-93 (2006).

${ }^{32}$ I. A. Brezovich, J. Duan, P. N. Pareek, J. Fiveash, and M. Ezekiel, "In vivo urethral dose measurements: A method to verify high dose rate prostate treatments," Med. Phys. 27, 2297-2301 (2000).

${ }^{33}$ J. E. Cygler, A. Saoudi, G. Perry, C. Morash, and C. E., "Feasibility study of using MOSFET detectors for in vivo dosimetry during permanent lowdose-rate prostate implants," Radiother. Oncol. 80, 296-301 (2006).

${ }^{34}$ E. J. Bloemen-van Gurp et al., "In vivo dosimetry using a linear MOSFETarray dosimeter to determine the urethra dose in ${ }^{125}$ I permanent prostate implants,” Int. J. Radiat. Oncol., Biol., Phys. 73, 314-321 (2009).

${ }^{35}$ C. E. Andersen, S. K. Nielsen, J. C. Lindegaard, and K. Tanderup, "Timeresolved in vivo luminescence dosimetry for online error detection in pulsed dose-rate brachytherapy," Med. Phys. 36, 5033-5043 (2009).

${ }^{36}$ G. Kertzscher, C. E. Andersen, F. A. Siebert, S. K. Nielsen, J. C. Lindegaard, and K. Tanderup, "Identifying afterloading PDR and HDR brachytherapy errors using real-time fiber-coupled $\mathrm{Al}_{2} \mathrm{O}_{3}: \mathrm{C}$ dosimetry and a novel statistical error decision criterion," Radiother. Oncol. 100, 456-462 (2011).

${ }^{37}$ D. O'Connell, C. A. Joslin, N. Howard, N. W. Ramsey, and W. E. Liversage, "The treatment of uterine carcinoma using the Cathetron. Part I. Technique," Br. J. Radiol. 40, 882-887 (1967).

${ }^{38}$ C. A. Joslin, C. W. Smith, and A. Mallik, "The treatment of cervix cancer using high activity ${ }^{60}$ Co sources," Br. J. Radiol. 45, 257-270 (1972).

${ }^{39}$ K. Schultka, B. Ciesielski, K. Serkies, T. Sawicki, Z. Tarnawska, and J. Jassem, "EPR/alanine dosimetry in LDR brachytherapy: A feasibility study," Radiat. Prot. Dosim. 120, 171-175 (2006).

${ }^{40}$ M. Allahverdi, M. Sarkhosh, M. Aghili, R. Jaberi, A. Adelnia, and G. Geraily, "Evaluation of treatment planning system of brachytherapy according to dose to the rectum delivered," Radiat. Prot. Dosim. 150, 312315 (2012).

${ }^{41}$ N. Suchowerska, M. Jackson, J. Lambert, Y. B. Yin, G. Hruby, and D. R. McKenzie, "Clinical trials of a urethral dose measurement system in brachytherapy using scintillation detectors," Int. J. Radiat. Oncol., Biol., Phys. 79, 609-615 (2011).
${ }^{42}$ J. A. Raffi et al., "Determination of exit skin dose for ${ }^{192}$ Ir intracavitary accelerated partial breast irradiation with thermoluminescent dosimeters," Med. Phys. 37, 2693-2702 (2010).

${ }^{43}$ R. A. Kinhikar et al., "Clinical application of a OneDose MOSFET for skin dose measurements during internal mammary chain irradiation with high dose rate brachytherapy in carcinoma of the breast," Phys. Med. Biol. 51, N263-N268 (2006)

${ }^{44}$ C. A. Mangold, A. Rijnders, D. Georg, L. E. Van, R. Pötter, and D. Huyskens, "Quality control in interstitial brachytherapy of the breast using pulsed dose rate: Treatment planning and dose delivery with an Ir192 afterloading system," Radiother. Oncol. 58, 43-51 (2001).

${ }^{45}$ M. J. Rivard et al., "Update of AAPM Task Group No. 43 Report: A revised AAPM protocol for brachytherapy dose calculations," Med. Phys. 31, 633-674 (2004).

${ }^{46} \mathrm{R}$. Bachelot-Narquin, "Measures taken by the French Health Minister to ensure safety in radiotherapy treatments," Safety in External Radiotherapy Treatments (ASN, Paris, 2009), Vol. 185, pp. 5-7 (available URL: http://www.conference-radiotherapy-asn.com).

${ }^{47} \mathrm{~F}$. Guedea et al., "Overview of brachytherapy resources in Europe: A survey of patterns of care study for brachytherapy in Europe," Radiother. Oncol. 82, 50-54 (2007).

${ }^{48}$ F. Guedea et al., "Overview of brachytherapy resources in Latin America: A patterns-of-care survey," Brachytherapy 10, 363-368 (2011).

${ }^{49}$ R. Pötter, L. E. Van, N. Gerstner, and A. Wambersie, "Survey of the use of the ICRU 38 in recording and reporting cervical cancer brachytherapy," Radiother. Oncol. 58, 11-18 (2001).

${ }^{50} \mathrm{~T}$. Toita et al., "Patterns of radiotherapy practice for patients with cervical cancer (1999-2001): Patterns of care study in Japan," Int. J. Radiat. Oncol., Biol., Phys. 70, 788-794 (2008).

${ }^{51}$ W. Small, Jr., B. Erickson, and F. Kwakwa, "American Brachytherapy Society survey regarding practice patterns of postoperative irradiation for endometrial cancer: Current status of vaginal brachytherapy," Int. J. Radiat. Oncol., Biol., Phys. 63, 1502-1507 (2005).

${ }^{52}$ K. S. Kapp, G. F. Stuecklschweiger, D. S. Kapp, and A. G. Hackl, "Dosimetry of intracavitary placements for uterine and cervical carcinoma: Results of orthogonal film, TLD, and CT-assisted techniques," Radiother. Oncol. 24, 137-146 (1992).

${ }^{53}$ D. W. O. Rogers, "General characteristics of radiation dosimeters and a terminology to describe them," in Clinical Dosimetry Measurements in Radiotherapy, edited by D. W. O. Rogers and J. Cygler (Medical Physics Publishing, Madison, 2009), pp. 137-146.

${ }^{54}$ J. F. Williamson and M. J. Rivard, "Quantitative dosimetry methods for brachytherapy," in Brachytherapy Physics, edited by B. Thomadsen, M. J. Rivard, and W. Butler (Medical Physics Publishing, Madison, WI, 2005).

${ }^{55}$ T. Nose et al., "In vivo dosimetry of high-dose-rate brachytherapy: Study on 61 head-and-neck cancer patients using radiophotoluminescence glass dosimeter,” Int. J. Radiat. Oncol., Biol., Phys. 61, 945-953 (2005).

${ }^{56} \mathrm{~T}$. Nose et al., "In vivo dosimetry of high-dose-rate interstitial brachytherapy in the pelvic region: Use of a radiophotoluminescence glass dosimeter for measurement of 1004 points in 66 patients with pelvic malignancy," Int. J. Radiat. Oncol., Biol., Phys. 70, 626-633 (2008).

${ }^{57}$ E. L. Seymour, S. J. Downes, G. B. Fogarty, M. A. Izard, and P. Metcalfe, "In vivo real-time dosimetric verification in high dose rate prostate brachytherapy," Med. Phys. 38, 4785-4794 (2011).

${ }^{58}$ J. Lambert, T. Nakano, S. Law, J. Elsey, D. R. McKenzie, and N. Suchowerska, "In vivo dosimeters for HDR brachytherapy: A comparison of a diamond detector, MOSFET, TLD, and scintillation detector," Med. Phys. 34, 1759-1765 (2007).

${ }^{59}$ A. S. Kirov, J. F. Williamson, A. S. Meigooni, and Y. Zhu, "TLD, diode and Monte Carlo dosimetry of an ${ }^{192}$ Ir source for high dose-rate brachytherapy," Phys. Med. Biol. 40, 2015-2036 (1995).

${ }^{60}$ M. Westermark, J. Arndt, B. Nilsson, and A. Brahme, "Comparative dosimetry in narrow high-energy photon beams," Phys. Med. Biol. 45, 685-702 (2000).

${ }^{61} \mathrm{G}$. Anagnostopoulos et al., "In vivo thermoluminescence dosimetry dose verification of transperineal ${ }^{192}$ Ir high-dose-rate brachytherapy using CTbased planning for the treatment of prostate cancer," Int. J. Radiat. Oncol., Biol., Phys. 57, 1183-1191 (2003).

${ }^{62}$ R. Das, W. Toye, T. Kron, S. Williams, and G. Duchesne, "Thermoluminescence dosimetry for in vivo verification of high dose rate brachytherapy for prostate cancer," Australas. Phys. Eng. Sci. Med. 30, 178-184 (2007). 
${ }^{63}$ W. Toye, R. Das, T. Kron, R. Franich, P. Johnston, and G. Duchesne, "An in vivo investigative protocol for HDR prostate brachytherapy using urethral and rectal thermoluminescence dosimetry," Radiother. Oncol. 91, 243-248 (2009).

${ }^{64}$ C. Waldhäusl, A. Wambersie, R. Potter, and D. Georg, "In-vivo dosimetry for gynaecological brachytherapy: Physical and clinical considerations," Radiother. Oncol. 77, 310-317 (2005).

${ }^{65}$ J. M. Fagerstrom, J. A. Micka, and L. A. DeWerd, "Response of an implantable MOSFET dosimeter to ${ }^{192}$ Ir HDR radiation," Med. Phys. 35, 5729-5737 (2008).

${ }^{66}$ C. E. Andersen, S. K. Nielsen, S. Greilich, J. Helt-Hansen, J. C. Lindegaard, and $\mathrm{K}$. Tanderup, "Characterization of a fiber-coupled $\mathrm{Al}_{2} \mathrm{O}_{3}: \mathrm{C}$ luminescence dosimetry system for online in vivo dose verification during ${ }^{192}$ Ir brachytherapy, Med. Phys. 36, 708-718 (2009).

${ }^{67}$ A. S. Beddar, T. R. Mackie, and F. H. Attix, "Water-equivalent plastic scintillation detectors for high-energy beam dosimetry: I. Physical characteristics and theoretical consideration," Phys. Med. Biol. 37, 1883-1900 (1992).

${ }^{68}$ J. Lambert, D. R. McKenzie, S. Law, J. Elsey, and N. Suchowerska, "A plastic scintillation dosimeter for high dose rate brachytherapy," Phys. Med. Biol. 51, 5505-5516 (2006).

${ }^{69}$ F. Therriault-Proulx, T. M. Briere, F. Mourtada, S. Aubin, S. Beddar, and L. Beaulieu, "A phantom study of an in vivo dosimetry system using plastic scintillation detectors for real-time verification of ${ }^{192}$ Ir HDR brachytherapy," Med. Phys. 38, 2542-2551 (2011).

${ }^{70}$ L. E. Cartwright, N. Suchowerska, Y. Yin, J. Lambert, M. Haque, and D. R. McKenzie, "Dose mapping of the rectal wall during brachytherapy with an array of scintillation dosimeters," Med. Phys. 37, 2247-2255 (2010).

${ }^{71} \mathrm{M}$. Anton et al., "In vivo dosimetry in the urethra using alanine/ESR during ${ }^{192}$ Ir HDR brachytherapy of prostate cancer: A phantom study," Phys. Med. Biol. 54, 2915-2931 (2009).

${ }^{72} \mathrm{~S}$. M. Hsu et al., "Clinical application of radiophotoluminescent glass dosimeter for dose verification of prostate HDR procedure," Med. Phys. 35, 5558-5564 (2008).

${ }^{73}$ A. Ismail et al., "Radiotherapy quality assurance by individualized in vivo dosimetry: State of the art," Cancer Radiother. 13, 182-189 (2009).

${ }^{74}$ J. E. Cygler, K. Tanderup, A. S. Beddar, and J. Perez-Calatayud, "In vivo dosimetry in brachytherapy," in Comprehensive Brachytherapy: Physical and Clinical Aspects, edited by J. Venselaar, D. Baltas, A. S. Meigooni, and P. Hoskin (Taylor \& Francis, London, 2012), pp. 379-397.

${ }^{75}$ D. W. O. Rogers and J. Cygler, Clinical Dosimetry Measurements in Radiotherapy (Medical Physics Publishing, Madison, 2009).

${ }^{76}$ A. Piermattei, L. Azario, G. Monaco, A. Soriani, and G. Arcovito, "p-type silicon detector for brachytherapy dosimetry," Med. Phys. 22, 835-839 (1995).

${ }^{77}$ M. Soubra, J. Cygler, and G. Mackay, "Evaluation of a dual bias dual metal oxide-silicon semiconductor field effect transistor detector as radiation dosimeter," Med. Phys. 21, 567-572 (1994).

${ }^{78}$ V. O. Zilio, O. P. Joneja, Y. Popowski, A. Rosenfeld, and R. Chawla, "Absolute depth-dose-rate measurements for an ${ }^{192}$ Ir HDR brachytherapy source in water using MOSFET detectors," Med. Phys. 33, 1532-1529 (2006).

${ }^{79}$ H. Niu, W. C. Hsi, J. C. Chu, M. C. Kirk, and E. Kouwenhoven, "Dosimetric characteristics of the Leipzig surface applicators used in the high dose rate brachy radiotherapy," Med. Phys. 31, 3372-3377 (2004).

${ }^{80}$ E. J. Bloemen-van Gurp et al., "In vivo dosimetry with a linear MOSFET array to evaluate the urethra dose during permanent implant brachytherapy using iodine-125," Int. J. Radiat. Oncol., Biol., Phys. 75, 1266-1272 (2009).

${ }^{81}$ A. Cherpak, J. Cygler, and G. Perry, "Real-time measurement of urethral dose and position using a RADPOS array during permanent seed implantation for prostate brachytherapy," Med. Phys. 38, 3577 (2011).

${ }^{82}$ A. Cherpak, W. Ding, A. Hallil, and J. E. Cygler, "Evaluation of a novel 4D in vivo dosimetry system," Med. Phys. 36, 1672-1679 (2009).
${ }^{83}$ B. Reniers, G. Landry, R. Eichner, A. Hallil, and F. Verhaegen, "In vivo dosimetry for gynaecological brachytherapy using a novel position sensitive radiation detector: Feasibility study," Med. Phys. 39, 1925-1935 (2012).

${ }^{84}$ M. Farahani, F. C. Eichmiller, and W. L. McLaughlin, "New method for shielding electron beams used for head and neck cancer treatment," Med. Phys. 20, 1237-1241 (1993).

${ }^{85}$ S. Olsson, E. S. Bergstrand, A. K. Carlsson, E. O. Hole, and E. Lund, "Radiation dose measurements with alanine/agarose gel and thin alanine films around a ${ }^{192}$ Ir brachytherapy source, using ESR spectroscopy," Phys. Med. Biol. 47, 1333-1356 (2002).

${ }^{86}$ C. S. Calcina, A. A. de, J. R. Rocha, F. C. Abrego, and O. Baffa, "Ir192 HDR transit dose and radial dose function determination using alanine/EPR dosimetry," Phys. Med. Biol. 50, 1109-1117 (2005).

${ }^{87}$ A. C. De, S. Onori, E. Petetti, A. Piermattei, and L. Azario, "Alanine/EPR dosimetry in brachytherapy,” Phys. Med. Biol. 44, 1181-1191 (1999).

${ }^{88}$ B. Ciesielski, K. Schultka, A. Kobierska, R. Nowak, and Z. PeimelStuglik, "In vivo alanine/EPR dosimetry in daily clinical practice: A feasibility study,” Int. J. Radiat. Oncol., Biol., Phys. 56, 899-905 (2003).

${ }^{89} \mathrm{~S}$. W. McKeever et al., "Recent advances in dosimetry using the optically stimulated luminescence of $\mathrm{Al}_{2} \mathrm{O}_{3}$ :C," Radiat. Prot. Dosim. 109, 269-276 (2004).

${ }^{90}$ E. G. Yukihara and S. W. McKeever, "Optically stimulated luminescence (OSL) dosimetry in medicine," Phys. Med. Biol. 53, R351-R379 (2008).

${ }^{91}$ G. Kertzscher, C. E. Andersen, J. Edmund, and K. Tanderup, "Stem signal suppression in fiber-coupled $\mathrm{Al}_{2} \mathrm{O}_{3}$ :C dosimetry for ${ }^{192} \mathrm{Ir}$ brachytherapy," Radiat. Meas. 46, 2020-2024 (2011).

${ }^{92}$ A. S. Beddar, T. R. Mackie, and F. H. Attix, "Water-equivalent plastic scintillation detectors for high-energy beam dosimetry: II. Properties and measurements," Phys. Med. Biol. 37, 1901-1913 (1992).

${ }^{93}$ A. S. Beddar, N. Suchowerska, and S. H. Law, "Plastic scintillation dosimetry for radiation therapy: Minimizing capture of Cerenkov radiation noise," Phys. Med. Biol. 49, 783-790 (2004).

${ }^{94}$ L. Archambault, A. S. Beddar, L. Gingras, R. Roy, and L. Beaulieu, "Measurement accuracy and Cerenkov removal for high performance, high spatial resolution scintillation dosimetry," Med. Phys. 33, 128-135 (2006).

${ }^{95}$ A. R. Beierholm, R. O. Ottosson, L. R. Lindvold, C. F. Behrens, and C. E. Andersen, "Characterizing a pulse-resolved dosimetry system for complex radiotherapy beams using organic scintillators," Phys. Med. Biol. 56, 3033-3045 (2011).

${ }^{96}$ L. Archambault et al., "Toward a real-time in vivo dosimetry system using plastic scintillation detectors," Int. J. Radiat. Oncol., Biol., Phys. 78, 280287 (2010).

${ }^{97} \mathrm{~S}$. Beddar, "On possible temperature dependence of plastic scintillator response," Med. Phys. 39, 6522 (2012).

${ }^{98}$ F. Therriault-Proulx, L. Archambault, L. Beaulieu, and S. Beddar, "Development of a novel multi-point plastic scintillation detector with a single optical transmission line for radiation dose measurement," Phys. Med. Biol. 57, 7147-7159 (2012).

${ }^{99}$ A. Cherpak, M. Serban, J. Seuntjens, and J. E. Cygler, "4D dose-position verification in radiation therapy using the RADPOS system in a deformable lung phantom," Med. Phys. 38, 179-187 (2011).

${ }^{100}$ A. J. Cherpak, J. E. Cygler, S. Andrusyk, J. Pantarotto, R. MacRae, and G. Perry, "Clinical use of a novel in vivo 4D monitoring system for simultaneous patient motion and dose measurements," Radiother. Oncol. 102, 290-296 (2012).

${ }^{101}$ L. N. McDermott et al., "3D in vivo dose verification of entire hypofractionated IMRT treatments using an EPID and cone-beam CT," Radiother. Oncol. 86, 35-42 (2008).

${ }^{102}$ J. Cunningham, M. Coffey, T. Knoos, and O. Holmberg, "Radiation oncology safety information system (ROSIS): Profiles of participants and the first 1074 incident reports," Radiother. Oncol. 97, 601-607 (2010).

${ }^{103}$ G. O. Sawakuchi, L. Archambault, A. Scullion, and J. E. Cygler, "Results of a survey to assess the current status of in-vivo dosimetry in Canada," Interactions: the Canadian Medical Physics Newsletter 58(1), 13-18, 2012. 